\title{
Association of DNA Repair Gene APE1 Asp148Glu Polymorphism with Breast Cancer Risk
}

\author{
Fatima AlMutairi, ${ }^{1}$ Akbar Ali Khan Pathan,, ${ }^{1,2}$ Mohammed Alanazi, ${ }^{1}$ Manal Shalaby, ${ }^{1,3}$ \\ Huda A. Alabdulkarim, ${ }^{4}$ Abdullah Alamri, ${ }^{1}$ Abdulrahman Al Naeem, ${ }^{5}$ Moammad Elrobh, \\ Jilani P. Shaik, ${ }^{1}$ Wajahatullah Khan, ${ }^{6}$ Zahid Khan, ${ }^{1}$ and Narasimha Reddy Parine ${ }^{1}$ \\ ${ }^{1}$ Genome Research Chair, Department of Biochemistry, College of Science, King Saud University, Riyadh 11451, Saudi Arabia \\ ${ }^{2}$ Integrated Gulf Biosystems, Riyadh 11391, Saudi Arabia \\ ${ }^{3}$ Genetic Engineering and Biotechnology Research Institute (GEBRI), City for Scientific Research and Technology Applications, \\ Alexandria 21934, Egypt \\ ${ }^{4}$ The Comprehensive Cancer Center, King Fahad Medical City, Riyadh 11525, Saudi Arabia \\ ${ }^{5}$ Department of Women's Imaging, King Fahad Medical City, Riyadh 11525, Saudi Arabia \\ ${ }^{6}$ Department of Basic Sciences, College of Science and Health Professions, King Saud Bin Abdul Aziz University for Health Sciences, \\ Riyadh 11426, Saudi Arabia
}

Correspondence should be addressed to Narasimha Reddy Parine; reddyparine@gmail.com

Received 13 February 2015; Revised 22 April 2015; Accepted 10 June 2015

Academic Editor: Andreas Pich

Copyright (C) 2015 Fatima AlMutairi et al. This is an open access article distributed under the Creative Commons Attribution License, which permits unrestricted use, distribution, and reproduction in any medium, provided the original work is properly cited.

Objective. The aim of this study was to investigate the role of APE1 Asp148Glu polymorphism in breast cancer progression in Saudi population. Methods. We examined the genetic variations (rs1130409) in the DNA base excision repair gene APE1 at codon 148 (Asp148Glu) and its association with breast cancer risk using genotypic assays and in silico structural as well as functional predictions. In silico structural analysis was performed with Asp148Glu allele and compared with the predicted native protein structure. The wild and mutant 3D structures of APE1 were compared and analyzed using solvent accessibility models for protein stability confirmation. Results. Genotypic analysis of APE1 (rs1130409) showed statistically significant association of Asp148Glu with elevated susceptibility to breast cancer. The in silico analysis results indicated that the nsSNP Asp148Glu may cause changes in the protein structure and is associated with breast cancer risk. Conclusion. Taken together, this is the first report that established that Asp148Glu variant has structural and functional effect on the APE1 and may play an important role in breast cancer progression in Saudi population.

\section{Introduction}

The incidence of breast cancer is highest among other cancers and is the main cause of cancer related deaths in Saudi women [1]. Compared to developed and Western region nations, the age adjusted rate for breast cancer in Saudi population is 4-5fold lower; however, the median age of diagnosis is 49 years, which is significantly lower compared to Western patients $[2,3]$. There is significant evidence that inadequate repair of DNA damage plays a major role in the progression of cancer and other human diseases [4]. Base excision repair (BER) is involved in repair of oxidative free radical induced
DNA lesions. Efficiency of (BER) is suggested to be a major determinant of breast cancer risk [5]. It is a key repair pathway that is accountable for conserving genome stability and consequently protecting from cancer and other diseases by repairing numerous lesions and strand breaks of DNA which are uninterruptedly caused by endogenous and exogenous mutagens [6]. When a single base is damaged, the BER pathway enzymes are responsible for recognizing and repairing the damaged base [7]. The first step in base excision repair pathway uses DNA glycosylases, to remove the damaged base to form an abasic or AP site by cleaving the N-glycosyl bond between the sugar and the base. Following the removal 
of the damaged base, apurinic/apyrimidinic endonuclease 1 (APE1) hydrolytically cleaves the phosphodiester backbone $5^{\prime}$ to the AP site, resulting in the formation of a $5^{\prime}$-deoxyribose phosphate $\left(5^{\prime}-\mathrm{dRP}\right)$ and a $3^{\prime}-\mathrm{OH}$ primer [8]. At this juncture, DNA polymerase then inserts a correct nucleotide and DNA ligases seal the repaired DNA strand. APE1 is a multifunctional enzyme which is located on chromosome 14q11.2q12 [9]. It exhibits DNA repair activity and has a role in the reductive activation of many transcription factors. These two functions are encoded by two different sites of APE1 enzyme. The $\mathrm{N}$-terminal region encodes the redox function and the C-terminal region encodes the repair function [10]. The DNA repair activity includes $3^{\prime}$-phosphodiesterase, $3^{\prime}$ phosphatase, and 3-5' -exonuclease activities [11]. To coordinate BER pathway APE1 interacts with PARP1, XRCC1, DNA polymerase $\beta$, and flap endonuclease 1 (FEN1) [12]. APE1 stimulates all these proteins individually. DNA polymerase then inserts a correct nucleotide and DNA ligases seal the repaired DNA strand. Mutations in this highly regulated mechanism can be caused by single nucleotide polymorphisms (SNPs), which will result in insufficient DNA repair which may enhance DNA lesions $[13,14]$. Mutations in APE1 gene may affect its function. If AP sites are not processed, mutagenesis and cellular cytotoxicity can result from blocked DNA replication machinery or from misincorporation of bases opposite the AP site [12,15]. The APE1 polymorphism at codon 148 has been previously reported to be associated with prostate cancer risk [14]. However, previous reports on the association between APE1 polymorphisms and cancer risk have provided inconsistent results [16-19]. Data pertaining the association of the APE1 polymorphisms with breast cancer risk are also inconsistent $[4,20]$. To the best of our knowledge, there is no published report on the association between APE1 SNP variant Asp148Glu (rs1130409) and breast cancer in Saudi population. Thus, this is the first study that investigates APE1 SNP Asp148Glu association with breast cancers using TaqMan assay in Saudi women. Additionally, in silico analyses were performed to determine the structural and functional consequence of Glu instead of Asp at codon 148 of APE1 protein.

\section{Materials and Methods}

2.1. Study Population. This study was a case-control study that included 100 Saudi female patients diagnosed with breast cancer at King Fahad Medical City Hospital, Riyadh, Saudi Arabia, along with 100 controls. Controls were age-matched and confirmed to be free of cancer and other diseases following thorough physical examinations. The demographic data of each patient and control were recorded (Table 1). Patients and controls were enrolled under King Khalid University Hospital Institutional Review Board approved protocol with written informed consent.

2.2. Genotyping. Genomic DNA was extracted from the blood samples of breast cancer cases and controls using QIAmp DNA blood Mini Kit (Qiagen, Valencia, CA) following the manufacturer's instructions. SNP rs1130409 in APE1 gene was genotyped using TaqMan allelic discrimination assay as described previously $[21,22]$. Ten percent of the
TABLE 1: Clinical characteristics of study subjects.

\begin{tabular}{lcc}
\hline Variable & Character & Number of samples \\
\hline Age (years) & $<48 />48$ & $47 / 53$ \\
Median age $\left(48 \pm 8.2^{*}\right)$ & ER+/ER- & $55 / 45$ \\
Estrogen receptor & PR+/PR- & $56 / 44$ \\
Progesterone receptor & HER+/HER- & $40 / 57$ \\
HER status &
\end{tabular}

${ }^{*}$ Median age with standard deviation.

samples were subjected to repeated analysis for verification of genotyping procedures.

2.3. Modeling of Mutant Structure. X-ray diffraction structure of APE1 $(2 \mathrm{O} 3 \mathrm{H})$ from PDB database was used as a reference to compare the wild-type (Asp148) protein structure with the predicted mutant structure (148Glu) and its solvent accessibility including secondary structures was modeled using molecular dynamics (MD) simulation. The best homology model for APE1 protein with Asp148Glu was selected using I-TASSER server [23]. The mutant APE1 Asp148Glu $3-\mathrm{D}$ structure was predicted using Modeller 9v10 [24]. The predicted model for mutant type protein was evaluated using ProSA-web [25].

2.4. Analyzing the Effects of Mutation on Protein Stability. Stability of the mutant protein is checked using prediction of Prediction of Protein Mutant Stability Changes (PoPMuSiC) [26]. The results were based on the selected $\Delta \Delta G$ values in $\mathrm{kcal} / \mathrm{mol}$ of the predicted APE1 Asp148Glu structure to evaluate the change in folding free energy after mutation $(\Delta \Delta G)$. Additionally, CUPSAT (Cologne University Protein Stability Analysis Tool) was also used to confirm the results [27].

2.5. The Effect of Mutant Residue 148Glu on APE1 Structure and Function. The presence of glutamate instead of aspartate at codon 148 of APE1 may affect the overall structure with a potential to alter its activity. The effect of Asp148Glu mutation was analyzed using Have yOur Protein Explained (HOPE) program [28] as described earlier by Alanazi et al. [29].

2.6. Molecular Dynamics Simulation. The effect of glutamate at codon 148 on APE1 was studied by comparing with threedimensional structure of APE1 protein present in the protein databank [PDB: 2O3H]. Structures deduced for APE1 harboring mutation Asp148Glu which is identified to be risk in Saudi breast cancer samples were utilized for the analyses. Biopolymer module in InsightII was used to substitute aspartate with glutamate residue at codon 148 from the fragment library and to add hydrogen atoms to both wild-type and mutated protein structures at pH 7.0 (Accelrys Inc., San Diego, CA). The molecular simulation program CHARMM was used to derive the force fields of both structures [30]. A sequence of energy minimization steps were performed as described by Alanazi et al. [31] on native and mutant protein structures by using InsightII/Discover (Accelrys Inc., San Diego, CA). Following energy minimization, protein structures were analyzed using Discovery Studio 2.5 (Accelrys Inc., San Diego, CA). 
TABLE 2: Genotype distribution of APE1 gene polymorphism in breast cancer cases and controls.

\begin{tabular}{|c|c|c|c|c|c|c|}
\hline Genotype & Cases & Controls & OR & $95 \% \mathrm{CI}$ & $\chi^{2}$ & $p$ value \\
\hline \multicolumn{7}{|l|}{ rs1130409 } \\
\hline Asp/Asp & $12(0.12)$ & $27(0.27)$ & Ref & & & \\
\hline Asp/Glu & $45(0.45)$ & $46(0.46)$ & 2.201 & $0.994-4.872$ & 3.87 & 0.04917 \\
\hline Glu/Glu & $43(0.43)$ & $27(0.27)$ & 3.583 & $1.558-8.243$ & 9.42 & 0.00215 \\
\hline Asp/Glu + Glu/Glu & $88(0.88)$ & $73(0.73)$ & 2.712 & $1.284-5.727$ & 7.17 & 0.00743 \\
\hline Asp & $69(0.345)$ & $100(0.5)$ & Ref & & & \\
\hline Glu & $131(0.65)$ & $100(0.5)$ & 1.899 & $1.270-2.839$ & 9.85 & 0.00170 \\
\hline
\end{tabular}

TABLE 3: Genotype frequencies of APE1 gene polymorphism in below 48 and above 48 years of age of breast cancer cases and controls.

\begin{tabular}{|c|c|c|c|c|c|c|}
\hline Genotype & Cases & Controls & OR & $95 \% \mathrm{CI}$ & $x^{2}$ & $p$ value \\
\hline rs1130409 & \multicolumn{2}{|c|}{ Below 48} & & & & \\
\hline Asp/Asp & $7(0.148)$ & $16(0.275)$ & Ref & & & \\
\hline Asp/Glu & $24(0.510)$ & $26(0.448)$ & 2.110 & $0.740-6.013$ & 1.99 & 0.15841 \\
\hline Glu/Glu & $16(0.340)$ & $16(0.275)$ & 2.286 & $0.741-7.051$ & 2.11 & 0.14678 \\
\hline Asp/Glu + Glu/Glu & $40(0.851)$ & $42(0.724)$ & 2.177 & $0.810-5.847$ & 2.44 & 0.11790 \\
\hline Asp & $38(0.404)$ & $58(0.5)$ & Ref & & & \\
\hline Glu & $56(0.595)$ & $58(0.5)$ & 1.474 & $0.851-2.553$ & 1.92 & 0.16607 \\
\hline rs1130409 & \multicolumn{2}{|c|}{ Above 48} & & & & \\
\hline Asp/Asp & $5(0.094)$ & $11(0.261)$ & Ref & & & \\
\hline Asp/Glu & $21(0.396)$ & $20(0.476)$ & 2.310 & $0.68-7.84$ & 1.85 & 0.17378 \\
\hline Glu/Glu & $27(0.509)$ & $11(0.261)$ & 5.400 & $1.52-19.20$ & 7.39 & 0.00656 \\
\hline Asp/Glu + Glu/Glu & $48(0.905)$ & $31(0.738)$ & 3.406 & $1.08-10.75$ & 4.70 & 0.03021 \\
\hline Asp & $31(0.292)$ & $42(0.5)$ & Ref & & & \\
\hline Glu & $75(0.707)$ & $42(0.5)$ & 2.419 & $1.33-4.40$ & 8.53 & 0.00349 \\
\hline
\end{tabular}

2.7. Statistical Analysis. Genotype and allelic frequencies were compared using Fisher's exact test (two-tailed) as described by Alanazi et al. [21] to estimate the $\chi^{2}$ test and odds ratios (OR) and $95 \%$ confidence intervals (CI) to know the variation between cancer cases and controls. All statistical analyses were performed using Statistical Package for the Social Sciences version 21.0 (SPSS Inc., Chicago, IL). The allele and genotype frequencies of APE1 (rs1130409) polymorphisms in the central region population of Saudi Arabia (CRS) were compared with some of the populations of the HapMap database, for example, Utah residents with northern and western European ancestry from the $\mathrm{CEPH}$ collection (CEU), Han Chinese in Beijing, China (CHB), Yoruba in Ibadan, Nigeria (YRI), Maasai in Kinyawa, Kenya (MKK), Japanese in Tokyo, Japan (JPT), Gujarati Indians in Houston, Texas (GIH), and Toscans in Italy (TSI) as described previously by Alanazi et al. [32]. Pairwise Chi-square $\left(\chi^{2}\right)$ tests were performed between the central region population of Saudi Arabia (CRS) and other populations using the allele frequencies in a $2 \times 2$ contingency table to study if the central region of Saudi population (CRS) shows significant differences compared to other populations.

\section{Results}

The present study examined the SNP rs1130409 (Asp148Glu) of APE1 gene in a total of 200 subjects. The distribution of the three genotypes as Asp/Asp, Asp/Glu, and Glu/Glu at codon 148 of the APE1 was significantly different between the controls and breast cancer patients $\left(\chi^{2}=9.44, \mathrm{df}=2\right.$, and $p=0.0089$ ). The genotype frequencies in breast cancer cases were $0.12,0.45$, and 0.43 for Asp/Asp, Asp/Glu, and Glu/Glu, respectively, whereas in healthy controls the frequencies of Asp/Asp, Asp/Glu, and Glu/Glu were 0.27, 0.46, and 0.27 , respectively (Table 2 ). The heterozygotes (Asp/Glu) and homozygote variant (Glu/Glu) showed significantly higher risk in breast cancer patients compared with the controls (Asp/Glu: $\mathrm{OR}=2.20, \chi^{2}=3.87$, and $p=0.0491$; Glu/Glu: OR: 3.58, $\chi^{2}=9.42$, and $\left.p=0.0021\right)$. The Glu allelic frequency of rs1130409 was higher (0.655) in the breast cancer patients than that in the control group $(0.50)(\mathrm{OR}=1.89$, $\chi^{2}=9.85$, and $p=0.0017$ ) (Table 2).

3.1. Effect of Age on the Association of APE1 SNP Asp148Glu with Breast Cancer. To examine the association of the SNPs with the age at the time of breast cancer diagnosis, we stratified the patients according to the median age at diagnosis (Table 1$)$ as $\leq 48(n=47)$ or $>48(n=53)$ years and compared them with age-matched controls. The analyses showed that the SNP rs1130409 did not have any association with breast cancers arising in women at or below 48 years of age (Table 3). However, APE1 codon 148 variant showed significant association with elderly breast cancer patients (>48 years) with Glu/Glu genotype as well as Glu allele posing higher risk (Table 3 ). 
TABLE 4: Genotype frequencies of APE1 gene polymorphism in breast cancer cases ER.

\begin{tabular}{|c|c|c|c|c|c|c|}
\hline Genotype & Cases & Controls & OR & $95 \% \mathrm{CI}$ & $\chi^{2}$ & $p$ value \\
\hline rs1130409 & ER +ve & & & & & \\
\hline Asp/Asp & $7(0.127)$ & $27(0.27)$ & Ref & & & \\
\hline Asp/Glu & $23(0.418)$ & $46(0.46)$ & 1.929 & 0.731-5.089 & 1.79 & 0.18065 \\
\hline Glu/Glu & $25(0.454)$ & $27(0.27)$ & 3.571 & $1.322-9.645$ & 6.65 & 0.00992 \\
\hline Asp/Glu + Glu/Glu & $48(0.872)$ & $73(0.73)$ & 2.536 & $1.023-6.286$ & 4.22 & 0.03992 \\
\hline Asp & $37(0.336)$ & $100(0.5)$ & Ref & & & \\
\hline Glu & $73(0.663)$ & $100(0.5)$ & 1.973 & $1.217-3.198$ & 7.71 & 0.00551 \\
\hline rs1130409 & ER -ve & & & & & \\
\hline Asp/Asp & $5(0.111)$ & $27(0.27)$ & Ref & & & \\
\hline Asp/Glu & $22(0.488)$ & $46(0.46)$ & 2.583 & $0.876-7.613$ & 3.09 & 0.07881 \\
\hline Glu/Glu & $18(0.4)$ & $27(0.27)$ & 3.600 & $1.168-11.09$ & 5.30 & 0.02127 \\
\hline Asp/Glu + Glu/Glu & $40(0.888)$ & $73(0.73)$ & 2.959 & $1.057-8.281$ & 4.56 & 0.03281 \\
\hline Asp & $32(0.355)$ & $100(0.5)$ & Ref & & & \\
\hline Glu & $58(0.644)$ & $100(0.5)$ & 1.812 & $1.085-3.027$ & 5.22 & 0.02230 \\
\hline
\end{tabular}

TABLE 5: Allele and genotype frequencies of APE1 rs1130409 (Asp148Glu) in central Saudi Arabia and other populations.

\begin{tabular}{|c|c|c|c|c|c|c|c|}
\hline \multirow[b]{2}{*}{ Population } & \multicolumn{3}{|c|}{ Genotype freq. (number) } & \multicolumn{2}{|c|}{ Allele frequency } & \multirow{2}{*}{$\begin{array}{l}\text { Pairwise } \chi^{2} \text { test value } \\
\text { between CRS \& other } \\
\text { populations }\end{array}$} & \multirow[b]{2}{*}{$p$ value } \\
\hline & $\begin{array}{c}\text { Asp/Asp freq. } \\
\text { (number) }\end{array}$ & $\begin{array}{l}\text { Asp/Glu freq. } \\
\text { (number) }\end{array}$ & $\begin{array}{l}\text { Glu/Glu freq. } \\
\text { (number) }\end{array}$ & $\begin{array}{l}\text { Wild type } \\
\text { Asp }\end{array}$ & $\begin{array}{c}\text { Variant } \\
\text { Glu }\end{array}$ & & \\
\hline $\mathrm{CEU}(n=226)$ & 0.319 & 0.407 & 0.274 & 0.52 & 0.48 & 0.13 & 0.71 \\
\hline $\mathrm{CHB}(n=84)$ & 0.214 & 0.476 & 0.310 & 0.45 & 0.55 & 0.41 & 0.51 \\
\hline $\mathrm{JPT}(n=170)$ & 0.094 & 0.494 & 0.412 & 0.34 & 0.66 & 6.61 & 0.01 \\
\hline $\mathrm{GIH}(n=176)$ & 0.114 & 0.341 & 0.545 & 0.28 & 0.72 & 12.86 & $0.0003^{*}$ \\
\hline YRI $(n=226)$ & 0.133 & 0.381 & 0.487 & 0.32 & 0.68 & 9.24 & $0.002^{*}$ \\
\hline $\operatorname{MKK}(n=286)$ & 0.098 & 0.524 & 0.378 & 0.36 & 0.64 & 6.05 & 0.01 \\
\hline TSI $(n=174)$ & 0.230 & 0.448 & 0.322 & 0.45 & 0.55 & 0.53 & 0.46 \\
\hline CRS $(n=100)$ & 0.27 & 0.46 & 0.27 & 0.50 & 0.50 & Ref & - \\
\hline
\end{tabular}

Population descriptions:

CEU: Utah residents with Northern and Western European ancestry from the CEPH collection.

CHB: Han Chinese in Beijing, China.

JPT: Japanese in Tokyo, Japan.

GIH: Gujarati Indians in Houston, Texas.

YRI: Yoruba in Ibadan, Nigeria.

MKK: Maasai in Kinyawa, Kenya.

TSI: Toscans in Italy.

CRS: Saudi population residing in Riyadh region of central Saudi Arabia.

* $p$ values significant after Bonferroni correction.

3.2. Effect of ER Status on the Association of APE1 SNP Asp148Glu with Breast Cancer. The association of breast cancer risk with the SNP variant rs1130409 based on the estrogen receptor (ER) status of the cancer patients was also investigated. The genotype distribution in the ER+ $(n=55)$ and ER- $(n=45)$ was independently compared with the genotypes in the control samples $(n=100)$ (Table 4$)$. APE1 variant rs1130409 showed higher risk in both ER+ and ERbreast cancer patients for Glu/Glu genotype and Glu alleles (Table 4).

3.3. Genotype and Allele Frequencies of APE1 Variant rs1130409 in Saudis and Other Populations. We compared the genotypic and allelic frequencies of the APE1 SNP rs1130409 in a normal healthy Saudi population with some other populations available in the HapMap database. The observed Asp/Asp, Asp/Glu, and Glu/Glu genotype frequencies in the Saudi population were $0.27,0.46$, and 0.27 , respectively (Table 5). Both the Asp (wild-type) and the Glu (variant) allele frequencies were similar and found to be 0.50 in Saudi population. The variant allele frequency ranged from 0.48 in CEU to 0.72 in GIH population. The CRS population frequencies differed significantly from GIH, YRI, and MKK populations based on the pairwise Chi-square $\left(\chi^{2}\right)$ tests (Table 5).

3.4. Effect of APE1 Asp148Glu Substitution on Protein Stability. Thermodynamic protein stability variations due to the replacement of Asp148 with 148Glu in the APE1 were predicted using PoPMusic. It revealed that the amino acid 


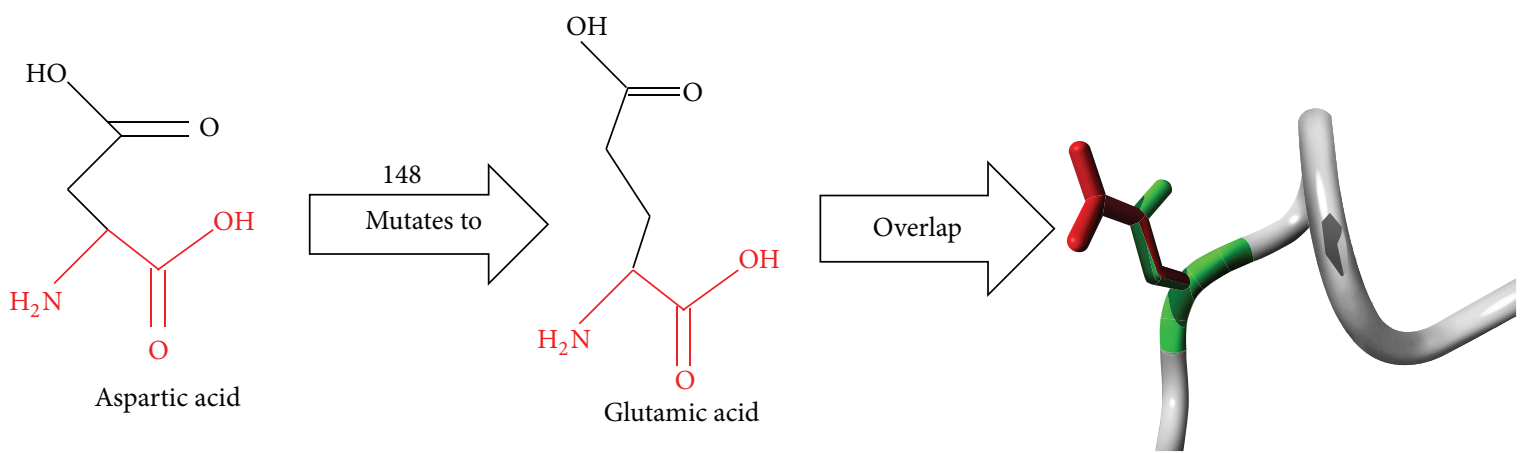

FIGURE 1: Schematic structure of the wild-type and mutant (Asp148Glu) amino acid.

alteration resulted in low levels of folding free energy $(\Delta \Delta G=$ $1.16 \mathrm{kcal} / \mathrm{mol}$ ) and caused structural destabilizing effects. Similar results were observed with CUPSAT as well. APE1 Asp148Glu exhibited unfavorable changes in torsion angles which influenced the overall stability of the protein. APE1 148Glu tertiary structure revealed significant variations due to protein folding in the mutated region between predicted and measured stability changes.

3.5. Effect of Mutant Residue 148Glu on APE1 Structure and Function. The mutant Glu residue at position 148 of APE1 protein was bigger than the wild-type Asp residue. Have yOur Protein Explained (HOPE) which collects and combines information from several webservers and databases was used to analyze the effect of Asp148Glu on APE1.

Domains. Asp148Glu residue is part of APE1 interpro domain named "Exodeoxyribonuclease III xth" (IPR004808; see http://www.ebi.ac.uk/interpro/entry/IPR004808;jsessionid= BEC787876CD492333C5F42D24F5AFD6B). This domain is annotated with the following Gene-Ontology (GO) terms to indicate its function: nuclease activity (GO: 0004518) and DNA repair (GO: 0006281; see http://www.ebi.ac.uk/ QuickGO/GTerm?id=GO:0006281). More broadly speaking, these Gene-Ontology annotations indicate that the domain has a function in hydrolase activity (GO: 0016787; see http:// www.ebi.ac.uk/QuickGO/GTerm?id=GO:0016787). This residue is part of an interpro domain named "Endonuclease/ exonuclease/phosphatase" (IPR005135; see http://www.ebi.ac .uk/interpro/entry/IPR005135; jsessionid=05F95A5D842E-

BA0A1048B8D753BB9705). Since the mutant residue was located in a domain which plays an important role primarily in the protein activity, it might disturb this function.

Conservation. The wild-type amino acid was not conserved at position 148 of APE1 protein and another residue type was observed more often at this position in other homologous sequences. This means that other homologous proteins exist with that other residue type more often than with the wildtype residue in the protein sequence. Therefore, the mutation is possibly damaging.

Amino Acid Properties. The wild and mutant type amino acids differ in size, where the mutant residue was found to be bigger, which may lead to altered structure.
3.6. MD Simulations of the Wild-Type and Mutant APE1. The 3D structure of APE1 (PDB ID: 2O3H) was already available from the protein database. This structure was used to examine the structural and functional effects of Asp148Glu substitutions in APE1. The structures of the wild-type amino acid aspartate and the risk conferring mutant amino acid glutamate were studied (Figure 1). The backbone was the same for the wild-type and the variant structures (shown in red color), whereas the side chain which was unique for wild and mutant type is shown in black color (Figure 1). Each amino acid depending on its side chain has its own specific size, charge, and hydrophobicity values. The mutant residue (Glu) due to the presence of an additional methylene group was larger in size than the wild-type (Asp) residue. When compared with other homologous protein sequences, the presence of the mutant glutamate reside at its respective position was not found to be conserved and hence could alter the structure and may have deleterious effect on APE1 function.

Substitution of Asp148 with 148Glu resulted in a slight worsening of ProSA-web $Z$-score, from -5.13 to -8.13 (Figure 2). The total energy deviation was -3 which may have a very unfavorable effect on the APE1 protein structure and function.

Molecular dynamics simulations were carried out using the APE1 structural information from the PDB database. The amino acid sequence and the open reading frame of the APE1 were submitted to I-TASSER program and, of the best five models that were generated, $2 \mathrm{O} 3 \mathrm{H}$ was selected based on the $C$-score $(-0.92)$, TM-score $(0.60 \pm 0.14)$, RMSD $(8.4 \pm$ $4.5 \AA$ ), number of decoys (4094), and cluster density (0.0756). The human APE1 (PDB ID: 2O3H) has 285 amino acid residues with three side chains (A, B, and C) (Figure 3(a)). The predicted structure with altered APE1 variant Asp148Glu was studied using Discovery Studio 2.5 and compared with the native structure (Figure 3(b)). The target amino acid at position 148 of APE1 protein was mutated from Asp to Glu and selected for the lowest energy rotamer conformations. The lowest potential energy state was achieved by atomic position arrangements using Steepest Descent (SD) energy minimization protocol for 200 steps and all water molecules were subsequently removed from the resulting structure. The Particle Mesh Ewald summation method was used for the estimation of the electrostatic energy with a distance cutoff 


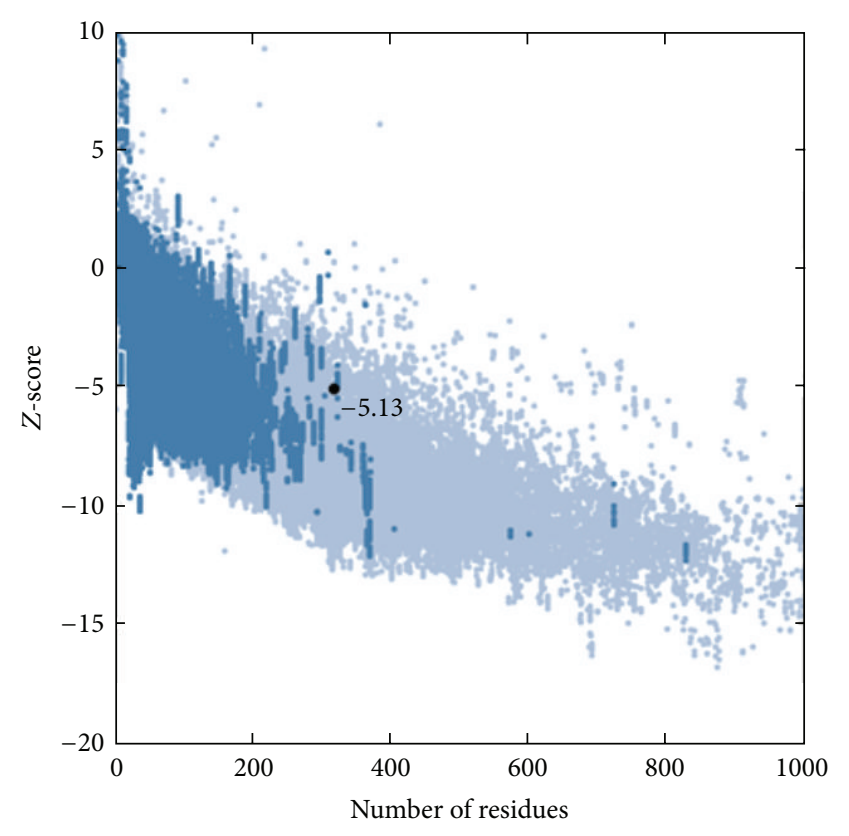

$\mathrm{X}$-ray

NMR

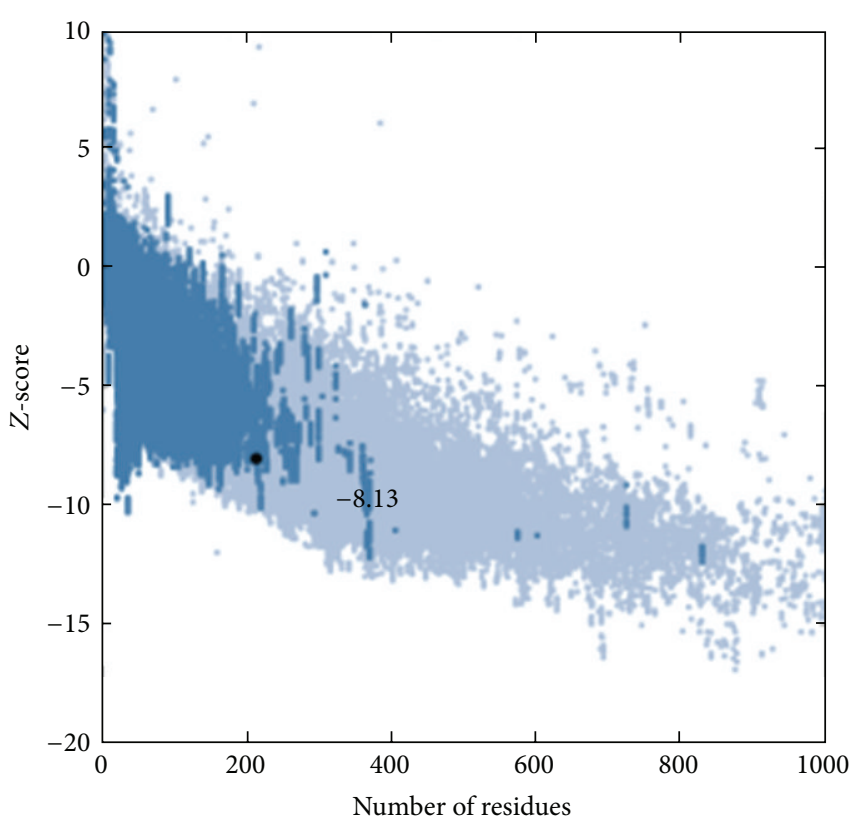

X-ray

NMR

FIgURE 2: Stability change of the mutant calculated by ProSA server.

of $10 \AA$. Similar procedure was followed for wild-type APE1 (PDB ID: 2O3H) structure to relax the crystal packing force to compare it with the mutant structure. Consequent to salvation, the resultant solvate showed successful accumulation of solvent around the predicted structure. The octahedral shapes of water box fitted fully to solvate the APE1 protein molecules with an edge distance of $10.0 \AA$ (Figure 3(c)). The wild and the mutant structures were superimposed to detect the effect of structural changes due to the mutation (Figure 3(b)). The structural and functional studies suggest that the variant allele (Asp148Glu) was localized in APE1 binding region; hence the mutation may play a significant role by altering its binding efficacy to its substrate and thus affecting the structural and functional properties of protein.

\section{Discussion and Conclusions}

The apurinic/apyrimidinic endonuclease (APE), $A P E 1$, is involved in the BER pathway [33]. Gene encoding APE1 has five exons with a $2.21 \mathrm{~kb}$ coverage on chromosome 14 (14q11.2-q12); when hydrolysed at the $3^{\prime}$ end it blocks DNA oxidisation, thus producing $3^{\prime}$-hydroxyl termini which is required for DNA repair during single- or double-strand breaks $[34,35]$. Imbalances in this tightly regulated process due to SNP may cause insufficient DNA repair mechanism and accumulate DNA breaks. In the present study we examined the role of APE1 variant Asp148Glu and breast cancer risk in Saudi females. The results showed that Asp148Glu variation may increase the risk of breast cancer by approximately 3.5-fold in Saudi patients (Table 2). Furthermore, the results also indicate that the Asp148Glu polymorphism was also associated with increased risk of breast cancer among subgroups of older subjects ( $>48$ years), in ER positive group as well as ER negative group (Tables 3 and 4). It is possible that the older individuals who showed higher risk association with breast cancer were more likely due to aging rather than direct genetic effects. It is more plausible that alteration in the APE1 gene may be more influential in early onset of breast cancer; however such an association was not observed in our younger group of patients (age $\leq 48$ years) probably due to small sample size.

This is the first report that deals with the APE1 variation Asp148Glu which significantly contributes to breast cancer susceptibility in Saudi females and suggests the importance of APE1 in breast carcinogenesis. The elevated risk of breast cancer in subjects with the APE1 alteration (Asp148Glu) can be attributed to the reduced APE1 activity in the DNA base excision repair pathway. Recent meta-analysis study [36] suggests that Asian populations are at higher risk of developing cancer than the non-Asian populations with APE1 Asp148Glu variant. Our results are in agreement with this observation and confirm that the Glu residue at position 148 of the APE1 confers significantly higher risk of breast cancer in Saudi females.

Saudi Arabian population has various tribes settled in different provinces for decades and these are usually recognized by their family names. The families residing in various provinces have been clustered based on their origin [32,37]. In the present study, the genotype and allele frequencies of rs1130409 (Asp148Glu) in a central region population of Saudi Arabia were observed and compared with various populations of HapMap database. The results showed that 


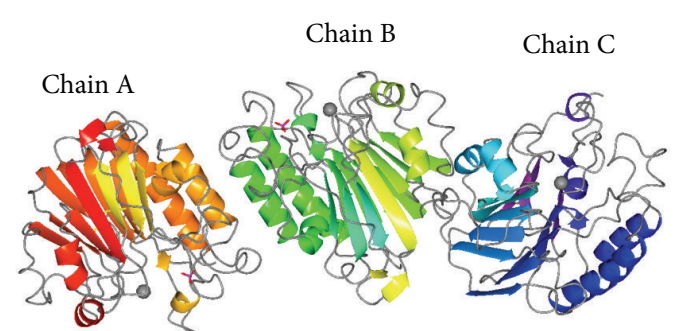

(a)

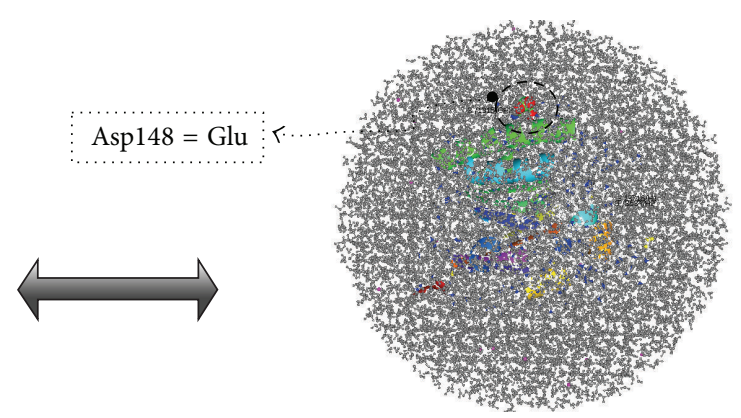

MD simulation spherical solvent boundary potential $0.51 \mathrm{M}$ ion/KCI ion (simulation)

(c)

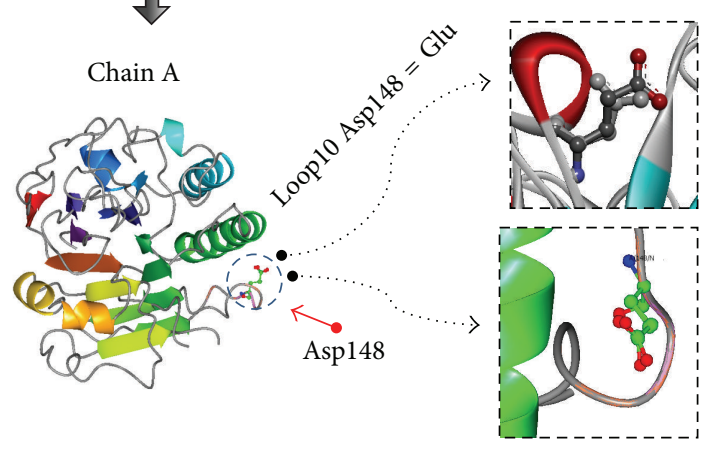

Structure superimposed with wild and mutant residues

(b)

FIGURE 3: (a) Ribbon diagram of APE1 protein showing all the three side chains A, B, and C. (b) APE1 structure superimposed with wild-type and mutant residue with mutation Asp148Glu enlarged. (c) Molecular dynamics (MD) simulation showing truncated octahedron boundary explicit water solvated and hydrogen atoms. The visual inspection also allows identifying the side chain of Glu residues involved in hydrogen bonding with the surrounding molecules.

the allelic frequencies for rs1130409 (Asp148Glu) were significantly different in the Saudi population compared to GIH, YRI, and MKK populations of HapMap database (Table 5). However, Chinese, Japanese, Italian, and northwestern European populations showed no significant difference in allelic frequencies for rs1130409 variant compared to the Saudi central region population. Hence, examining the SNP variant in other populations probably will not yield similar results, although APE1 (Asp148Glu) has previously been reported to be associated with breast cancer risk in Asian and European populations based on meta-analysis results.

We also evaluated the effect of Asp148Glu mutation on APE1 protein structure. Molecular dynamics methods using simulations in explicit solvent conditions were applied for investigating the wild and mutant amino acids and variation in APE1 protein dynamics and stability due to Asp148Glu variation. The energy minimization studies of the wild-type protein (Asp148) and the mutant type (148Glu) structures revealed that there was energy deviation due to Asp148Glu mutation $(-3 \mathrm{kcal} / \mathrm{mol})$. The mutant APE1 (Asp148Glu) structure stability based on thermodynamic changes was also detected using linear mixture of statistical potentials. Protein stability estimation using PoPMusic and
CUPSAT revealed that variant Asp148Glu caused structural destabilizing effects on the APE1 protein structure. Structural and functional analysis of the wild-type and variant APE1 revealed numerous multimer contacts including the one associated with nuclease (GO: 0004518) and hydrolase activity (GO: 0016787). APE1 variant Asp148Glu was present in an interpro domain exonuclease/endonuclease/phosphatase (IPR005135) which is responsible for the main activity of the protein; therefore any mutation in this region may affect the function of the protein.

Along with DNA repair activity, APE1 has another major function, which is also known as the redox effector factor 1 (Ref-1) [11]. APE1/Ref-1 reductively activates transcription factors including c-Jun, activator protein-1 (AP-1), nuclear factor kappa B (NF- $\kappa \mathrm{B})$, the tumor-suppressor protein $\mathrm{p} 53$, hypoxia-inducible factor 1a (HIF-1a), and paired box gene 8 , which are involved in various cellular processes such as cell survival, growth signaling, and inflammatory pathways [3841]. APE1 was also identified as a direct trans-acting factor for repressing genes by binding to the negative calcium-response element in their promoters. APE1/Ref-1 dysregulation has been reported to be associated with several diseases such as neurodegenerative [42] and cardiovascular diseases [43] 
and with various human cancers [44, 45]. APE1/Ref-1 may have a role in cancer progression via its ability to increase DNA repair and antiapoptotic, inflammatory, and growthpromoting activities [46]. APE1 gene polymorphisms may lead to amino acid substitutions, which may result in alterations of the functions of APE1/Ref-1.146. Our results support most of the previous studies which stated that Asp148Glu (T/G, codon 148, exon 5, and Asp to Glu) has a role in cancer development and progression. APE1 Asp148Glu mutation is reported to be found among different populations with high frequency and has been associated with various tumors [4749].

Overall our study has several key findings based on genetic and computational methods to implicate APE1 in the development of breast cancer. The strength of this study is that cancer cases and normal control samples were collected from the central region of Saudi Arabia and errors in genotyping were evaded by replicating select samples for random confirmation of the results. Limitations of these association analyses include the fact that the breast cancer cases were stratified for certain variables such as age at cancer diagnosis and ER status to assess its possible effect; however, the sample size is small and limited to the central region of Saudi population. Hence, in future studies the present data should be validated with larger number of samples as well as in other ethnic groups living in Saudi Arabia.

In conclusion, this is the first study showing an association between the APE1 Asp148Glu genotypes and increased risk of breast cancer in Saudi patients. Genotyping and in silico prediction based on $\mathrm{MD}$ simulation results suggest that the APE1 Asp148Glu variant may alter the BER pathway activity, hence probably contributing to breast carcinogenesis as its dysfunction may play a major role in the development of breast carcinoma. Additional detailed functional as well as association studies with larger sample size are needed to elucidate the role of APE1 polymorphism and associated breast cancer risk in Saudi population.

\section{Abbreviations}

APE1: $\quad$ Apurinic/apyrimidinic endonuclease 1

MD simulation: Molecular dynamics simulation

PoPMuSiC: $\quad$ Prediction of Protein Mutant Stability Changes

CUPSAT: $\quad$ Cologne University Protein Stability Analysis Tool

HOPE: $\quad$ Have yOur Protein Explained.

\section{Conflict of Interests}

The authors declare that they have no competing interests.

\section{Authors' Contribution}

Narasimha Reddy Parine, Mohammed Alanazi, and Manal Shalaby conceived and designed the experiments. Fatima AlMutairi, Akbar Ali Khan Pathan, Narasimha Reddy Parine, and Jilani P. Shaik performed the experiments. Narasimha
Reddy Parine, Akbar Ali Khan Pathan, Fatima AlMutairi, and Wajahatullah Khan analyzed the data. Narasimha Reddy Parine, Zahid Khan, Wajahatullah Khan, Mohammed Alanazi, and Fatima AlMutairi wrote the paper. Sample collection and clinicopathological data were performed by Huda A. Alabdulkarim, and Abdulrahman Al Naeem. Narasimha Reddy Parine, Fatima AlMutairi, Akbar Ali Khan Pathan, and Mohammed Alanazi interpreted the data. Fatima AlMutairi and Akbar Ali Khan Pathan contributed equally.

\section{Acknowledgment}

This project was funded by the National Plan for Science, Technology and Innovation (MAARIFAH), King Abdulaziz City for Science and Technology, Kingdom of Saudi Arabia, Award no. 12-MED2622-02.

\section{References}

[1] J. Ferlay, I. Soerjomataram, M. Ervik et al., "GLOBOCAN 2012 v1.0, cancer incidence and mortality worldwide," IARC Cancer Base 11, International Agency for Research on Cancer, Lyon, France, 2013, http://globocan.iarc.fr.

[2] Saudi National Cancer Registry, Cancer Incidence and Survival Report Saudi Arabia 2007, Saudi National Cancer Registry, Riyadh, Saudi Arabia, 2011.

[3] W. F. Anderson, B. E. Chen, I. Jatoi, and P. S. Rosenberg, "Effects of estrogen receptor expression and histopathology on annual hazard rates of death from breast cancer," Breast Cancer Research and Treatment, vol. 100, no. 1, pp. 121-126, 2006.

[4] S. Sangrajrang, P. Schmezer, I. Burkholder et al., "Polymorphisms in three base excision repair genes and breast cancer risk in Thai women," Breast Cancer Research and Treatment, vol. 111, no. 2, pp. 279-288, 2008.

[5] L. Gros, M. K. Saparbaev, and J. Laval, "Enzymology of the repair of free radicals-induced DNA damage," Oncogene, vol. 21, no. 58, pp. 8905-8925, 2002.

[6] G. L. Dianov and U. Hübscher, "Mammalian base excision repair: the forgotten archangel," Nucleic Acids Research, vol. 41, no. 6, pp. 3483-3490, 2013.

[7] S. Mitra, T. Izumi, I. Boldogh, K. K. Bhakat, J. W. Hill, and T. K. Hazra, "Choreography of oxidative damage repair in mammalian genomes," Free Radical Biology and Medicine, vol. 33, no. 1, pp. 15-28, 2002.

[8] P. W. Doetsch and R. P. Cunningham, "The enzymology of apurinic/apyrimidinic endonucleases," Mutation Research/ DNA Repair, vol. 236, no. 2-3, pp. 173-201, 1990.

[9] B. Demple and L. Harrison, "Repair of oxidative damage to DNA: enzymology and biology," Annual Review of Biochemistry, vol. 63, pp. 915-948, 1994.

[10] G. Barzilay and I. D. Hickson, "Structure and function of apurinic/apyrimidinic endonucleases," BioEssays, vol. 17, no. 8, pp. 713-719, 1995.

[11] K. K. Bhakat, A. K. Mantha, and S. Mitra, "Transcriptional regulatory functions of mammalian AP-endonuclease (APE1/Ref1), an essential multifunctional protein," Antioxidants \& Redox Signaling, vol. 11, no. 3, pp. 621-637, 2009.

[12] S. Thakur, B. Sarkar, R. P. Cholia, N. Gautam, M. Dhiman, and A. K. Mantha, "APE1/Ref-1 as an emerging therapeutic target for various human diseases: phytochemical modulation of its 
functions," Experimental and Molecular Medicine, vol. 46, no. 7, article e106, 2014.

[13] H. W. Mohrenweiser, D. M. Wilson III, and I. M. Jones, "Challenges and complexities in estimating both the functional impact and the disease risk associated with the extensive genetic variation in human DNA repair genes," Mutation Research/Fundamental and Molecular Mechanisms of Mutagenesis, vol. 526, no. 1-2, pp. 93-125, 2003.

[14] A. Memisoglu and L. Samson, "Contribution of base excision repair, nucleotide excision repair, and DNA recombination to alkylation resistance of the fission yeast Schizosaccharomyces pombe," Journal of Bacteriology, vol. 182, no. 8, pp. 2104-2112, 2000.

[15] B. A. Manvilla, O. Wauchope, K. L. Seley-Radtke, and A. C. Drohat, "NMR studies reveal an unexpected binding site for a redox inhibitor of AP endonuclease 1," Biochemistry, vol. 50, no. 48, pp. 10540-10549, 2011.

[16] L. Chen, C. B. Ambrosone, J. Lee, T. A. Sellers, J. Pow-Sang, and J. Y. Park, "Association between polymorphisms in the DNA repair genes $X R C C 1$ and $A P E 1$, and the risk of prostate cancer in white and black Americans," The Journal of Urology, vol. 175, no. 1, pp. 108-112, 2006.

[17] J. Baute and A. Depicker, "Base excision repair and its role in maintaining genome stability," Critical Reviews in Biochemistry and Molecular Biology, vol. 43, no. 4, pp. 239-276, 2008.

[18] Z. Li, W. Guan, M.-X. Li et al., "Genetic polymorphism of DNA base-excision repair genes (APE1, OGG1 and XRCC1) and their correlation with risk of lung cancer in a Chinese population," Archives of Medical Research, vol. 42, no. 3, pp. 226-234, 2011.

[19] R. D. Mittal, R. K. Mandal, and R. Gangwar, "Base excision repair pathway genes polymorphism in prostate and bladder cancer risk in North Indian population," Mechanisms of Ageing and Development, vol. 133, no. 4, pp. 127-132, 2012.

[20] T. Sliwinski, P. Ziemba, Z. Morawiec, M. Kowalski, M. Zadrozny, and J. Blasiak, "Polymorphisms of the DNA polymerase beta gene in breast cancer," Breast Cancer Research and Treatment, vol. 103, no. 2, pp. 161-166, 2007.

[21] M. S. Alanazi, N. R. Parine, J. P. Shaik, H. A. Alabdulkarim, S. A. Ajaj, and Z. Khan, "Association of single nucleotide polymorphisms in Wnt signaling pathway genes with breast cancer in Saudi patients," PLoS ONE, vol. 8, no. 3, Article ID e59555, 2013.

[22] K. J. Livak, "Allelic discrimination using fluorogenic probes and the 5 ' nuclease assay," Genetic Analysis: Biomolecular Engineering, vol. 14, no. 5-6, pp. 143-149, 1999.

[23] A. Roy, A. Kucukural, and Y. Zhang, "I-TASSER: a unified platform for automated protein structure and function prediction," Nature Protocols, vol. 5, no. 4, pp. 725-738, 2010.

[24] A. Sali, L. Potterton, F. Yuan, H. Van Vlijmen, and M. Karplus, "Evaluation of comparative protein modeling by MODELLER," Proteins: Structure, Function and Genetics, vol. 23, no. 3, pp. 318326, 1995.

[25] M. Wiederstein and M. J. Sippl, "ProSA-web: interactive web service for the recognition of errors in three-dimensional structures of proteins," Nucleic Acids Research, vol. 35, no. 2, pp. W407-W410, 2007.

[26] Y. Dehouck, J. M. Kwasigroch, D. Gilis, and M. Rooman, "PoPMuSiC 2.1: a web server for the estimation of protein stability changes upon mutation and sequence optimality," BMC Bioinformatics, vol. 12, article 151, 2011.

[27] V. Parthiban, M. M. Gromiha, and D. Schomburg, "CUPSAT: prediction of protein stability upon point mutations," Nucleic Acids Research, vol. 34, pp. W239-W242, 2006.
[28] H. Venselaar, T. A. H. te Beek, R. K. P. Kuipers, M. L. Hekkelman, and G. Vriend, "Protein structure analysis of mutations causing inheritable diseases. An e-Science approach with life scientist friendly interfaces," BMC Bioinformatics, vol. 11, article 548, 2010.

[29] M. Alanazi, A. A. Pathan, Z. Abduljaleel et al., "Association between PARP-1 V762A polymorphism and breast cancer susceptibility in Saudi population," PLoS ONE, vol. 8, no. 12, Article ID e85541, 2013.

[30] B. R. Brooks, C. L. Brooks III, A. D. Mackerell Jr. et al., "CHARMM: the biomolecular simulation program," Journal of Computational Chemistry, vol. 30, no. 10, pp. 1545-1614, 2009.

[31] M. Alanazi, A. S. Al-Arfaj, Z. Abduljaleel et al., "Novel hypoxanthine guanine phosphoribosyltransferase gene mutations in Saudi Arabian hyperuricemia patients," BioMed Research International, vol. 2014, Article ID 290325, 12 pages, 2014.

[32] M. Alanazi, A. A. K. Pathan, S. A. Ajaj et al., "Dna repair genes XRCC1, XRCC3, XPD, AND OGG1 polymorphisms among the central region population of Saudi Arabia," Biological Research, vol. 46, no. 2, pp. 161-167, 2013.

[33] R. D. Wood, M. Mitchell, J. Sgouros, and T. Lindahl, "Human DNA repair genes," Science, vol. 291, no. 5507, pp. 1284-1289, 2001.

[34] T. Izumi, T. K. Hazra, I. Boldogh et al., "Requirement for human AP endonuclease 1 for repair of 3'-blocking damage at DNA single-strand breaks induced by reactive oxygen species," Carcinogenesis, vol. 21, no. 7, pp. 1329-1334, 2000.

[35] M. R. Kelley, DNA Repair in Cancer Therapy: Molecular Targets and Clinical Applications, Academic Press, 2012.

[36] Z. Zhao, C. Liu, Y. Zeng et al., "The association between the APE1 Asp148Glu polymorphism and breast cancer susceptibility: a meta-analysis based on case-control studies," Tumor Biology, vol. 35, no. 5, pp. 4727-4734, 2014.

[37] M. A. F. El-Hazmi, A. R. Al-Swailem, A. S. Warsy, A. M. AlSwailem, R. Sulaimani, and A. A. Al-Meshari, "Consanguinity among the Saudi Arabian population," Journal of Medical Genetics, vol. 32, no. 8, pp. 623-626, 1995.

[38] M. Ema, K. Hirota, J. Mimura et al., "Molecular mechanisms of transcription activation by HLF and HIFlalpha in response to hypoxia: their stabilization and redox signal-induced interaction with CBP/p300," The EMBO Journal, vol. 18, no. 7, pp. 1905-1914, 1999.

[39] C. Gaiddon, N. C. Moorthy, and C. Prives, "Ref-1 regulates the transactivation and pro-apoptotic functions of $\mathrm{p} 53$ in vivo," The EMBO Journal, vol. 18, no. 20, pp. 5609-5621, 1999.

[40] G. Tell, L. Pellizzari, D. Cimarosti, C. Pucillo, and G. Damante, "Ref-1 controls pax-8 DNA-binding activity," Biochemical and Biophysical Research Communications, vol. 252, no. 1, pp. 178183, 1998.

[41] S. Xanthoudakis, G. Miao, F. Wang, Y.-C. E. Pan, and T. Curran, "Redox activation of Fos-Jun DNA binding activity is mediated by a DNA repair enzyme," The EMBO Journal, vol. 11, no. 9, pp. 3323-3335, 1992.

[42] A. Y. Shaikh and L. J. Martin, "DNA base-excision repair enzyme apurinic/apyrimidinic endonuclease/redox factor-1 is increased and competent in the brain and spinal cord of individuals with amyotrophic lateral sclerosis," NeuroMolecular Medicine, vol. 2, no. 1, pp. 47-60, 2002.

[43] S. H. Song, E. J. Cho, M. S. Park et al., "Redox regulating protein APE1/Ref-1 expression is increased in abdominal aortic coarctation-induced hypertension rats," Journal of the Korean Society of Hypertension, vol. 18, no. 3, pp. 126-135, 2012. 
[44] A. Al-Attar, L. Gossage, K. R. Fareed et al., "Human apurinic/ apyrimidinic endonuclease (APE1) is a prognostic factor in ovarian, gastro-oesophageal and pancreatico-biliary cancers," British Journal of Cancer, vol. 102, no. 4, pp. 704-709, 2010.

[45] V. Di Maso, C. Avellini, L. S. Crocè et al., "Subcellular localization of APE1/Ref-1 in human hepatocellular carcinoma: possible prognostic significance," Molecular Medicine, vol. 13, pp. 89-96, 2007.

[46] G. Tell, F. Quadrifoglio, C. Tiribelli, and M. R. Kelley, "The many functions of APE1/Ref-1: not only a DNA repair enzyme," Antioxidants and Redox Signaling, vol. 11, no. 3, pp. 601-619, 2009.

[47] E. Canbay, B. Agachan, M. Gulluoglu et al., "Possible associations of APE1 polymorphism with susceptibility and HOGG1 polymorphism with prognosis in gastric cancer," Anticancer Research, vol. 30, no. 4, pp. 1359-1364, 2010.

[48] C. Liu, Q. Yin, L. Li, Y.-Z. Zhuang, X. Zu, and Y. Wang, "APE1 Asp148Glu gene polymorphism and bladder cancer risk: a meta-analysis," Molecular Biology Reports, vol. 40, no. 1, pp. 171-176, 2013.

[49] M. Pieretti, N. H. Khattar, and S. A. Smith, "Common polymorphisms and somatic mutations in human base excision repair genes in ovarian and endometrial cancers," Mutation ResearchMutation Research Genomics, vol. 432, no. 3-4, pp. 53-59, 2001. 


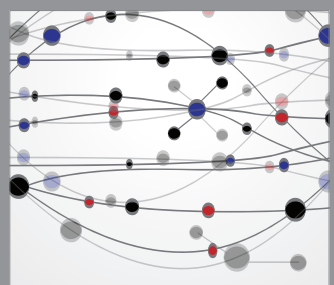

The Scientific World Journal
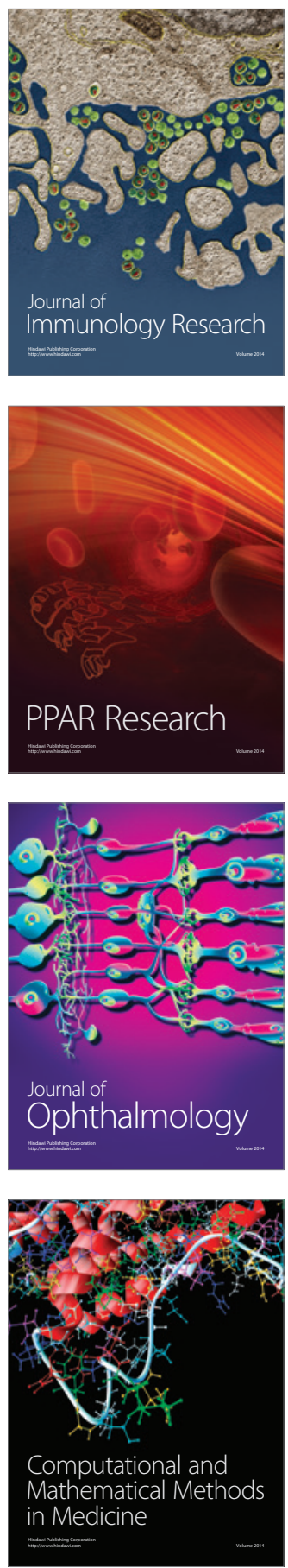

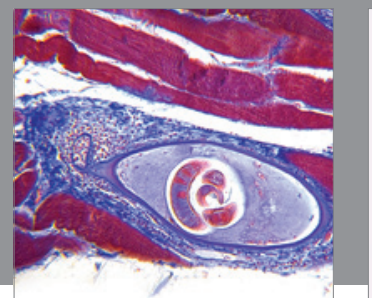

Gastroenterology

Research and Practice
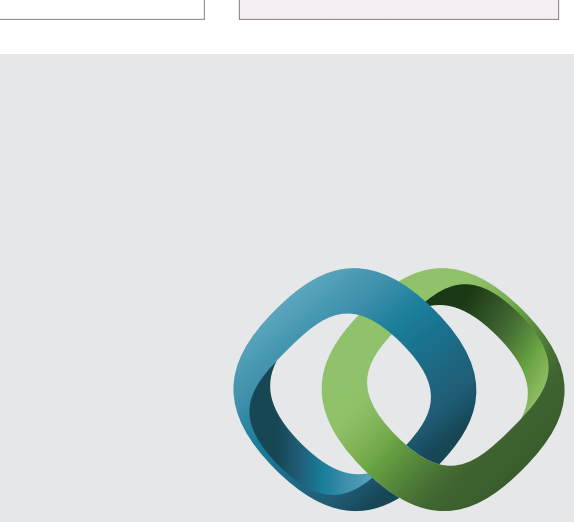

\section{Hindawi}

Submit your manuscripts at

http://www.hindawi.com
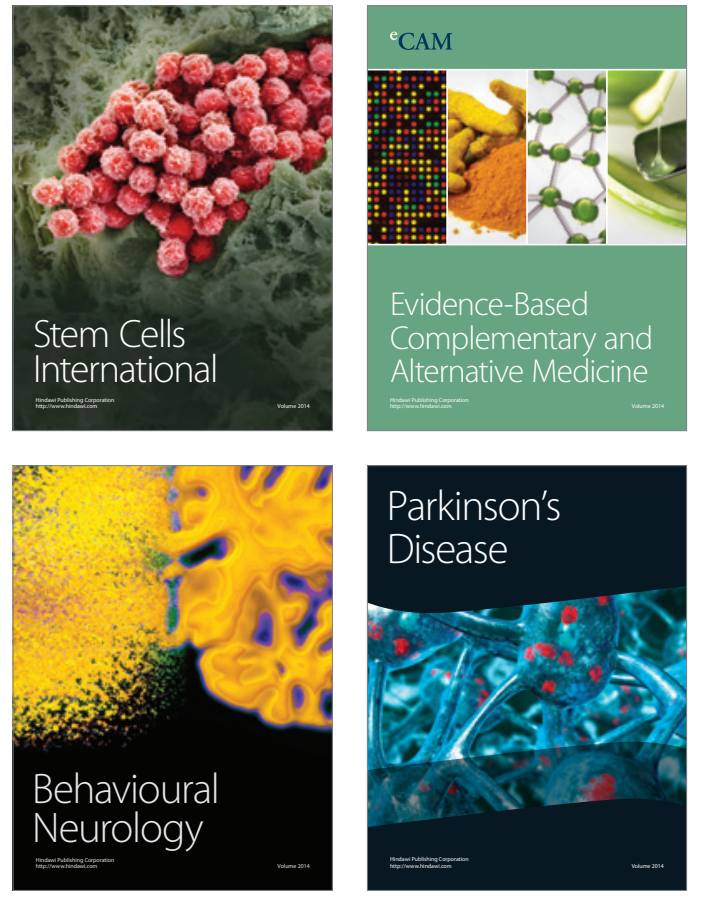
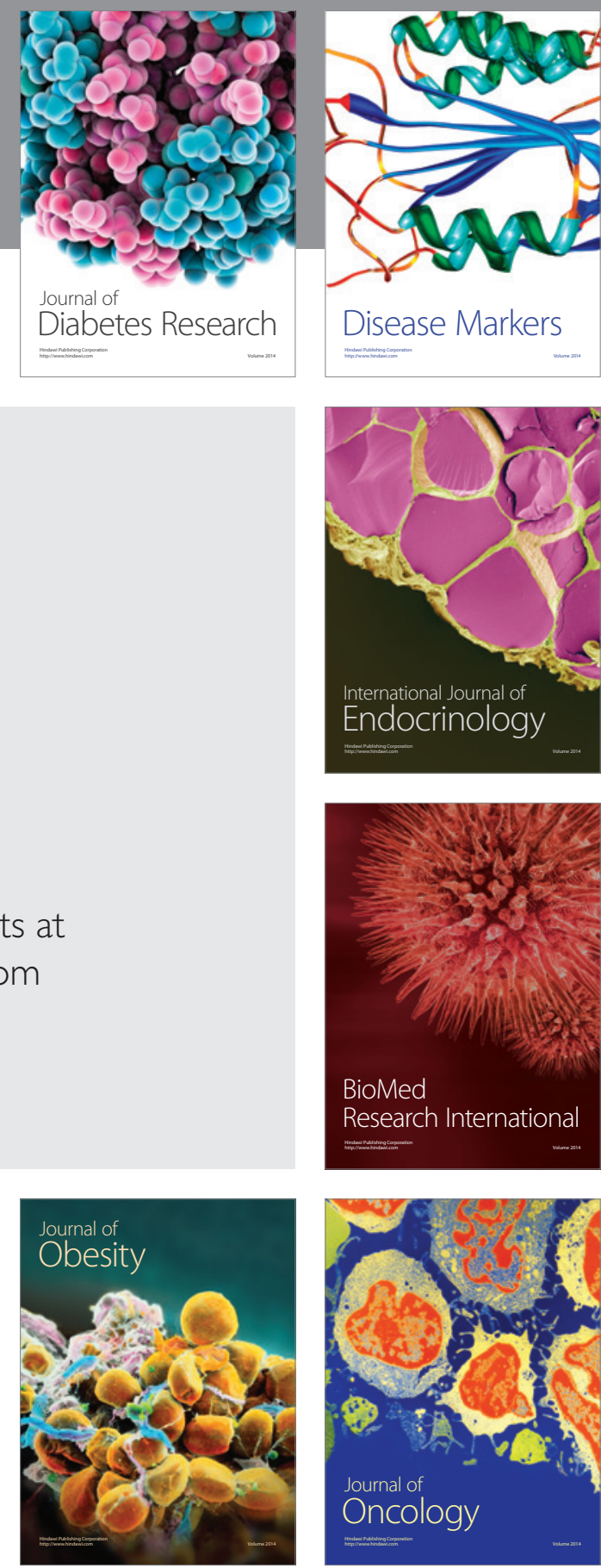

Disease Markers
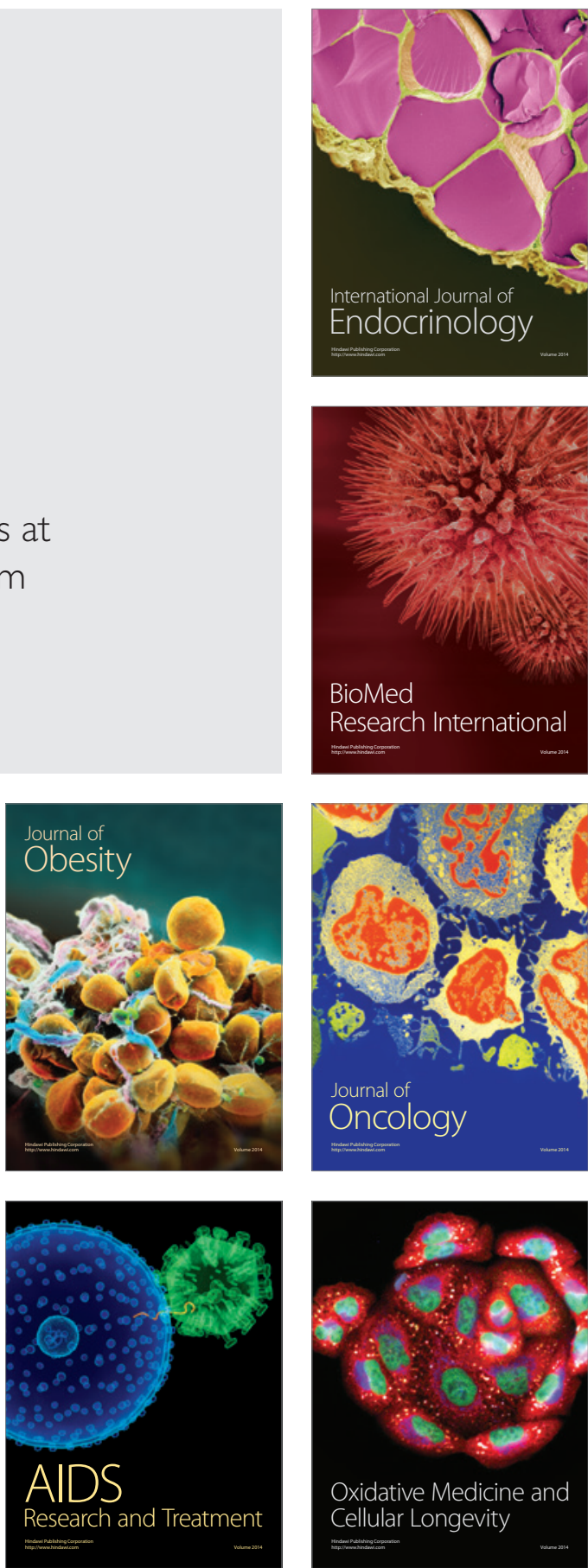